\title{
Molecular-dynamics study of self-interstitials in silicon
}

\author{
Inder P. Batra and Farid F. Abraham \\ IBM Almaden Research Center K33-801, 650 Harry Road, San Jose, California 95120-6099 \\ S. Ciraci \\ Department of Physics, Bilkent University, Ankara, Turkey
}

(Received 7 November 1986)

\begin{abstract}
Results of a molecular-dynamics computer simulation are presented for atomic relaxations and relaxation energies for self-interstitials in a silicon crystal. The Stillinger-Weber model potential containing two- and three-body terms is used and is expected to be more realistic than a simple Keating potential. The host crystal is represented by a cluster of 800 atoms, and the additional silicon atom was embedded in various interstitial sites near the center. The whole assembly was then periodically continued to fill the entire space. It is found that significant atomic relaxations occur in a shell of a radius $\sim 11$ a.u. and decay exponentially. In fact the relaxation is oscillatory in nature and also nonuniform within some shells. The calculated formation energies of vacancy and self-interstitials at equilibrium show trends which are in agreement with the self-consistent field total-energy calculations. These energy values are also in agreement with the known self-diffusion activation energy. From calculated formation energy values, we are able to draw the conclusion that the tetrahedralsite interstitial can be most readily formed. The hexagonal-site interstitial, on the other hand, is most repulsive. The migration from tetrahedral to dumbbell interstitial site appears to be most favorable.
\end{abstract}

\section{INTRODUCTION}

Silicon atoms located in noncrystallographic atomic positions in a Si crystal are called self-interstitials. The important features of the interstitials at low and high temperatures were already recognized more than a decade ago. ${ }^{1-4}$ It was known that at low temperature $(\sim 4 \mathrm{~K})$ the self-interstitials migrate with very high mobility. The lack of the phonon density as a source of driving force for the motion of the self-interstitials led one to seek for an athermal mechanism of migration. It was proposed that at very low temperature, interstitials should gain energy by capturing electrons in nonequilibrium states. ${ }^{1}$ At higher temperatures, the nature of defect primarily responsible for mediating self-diffusion has been widely debated. It was argued that at intermediate temperature the dumbbell (formed by replacing a single host atom with a pair of Si atoms) interstitial dominates the self-diffusion, whereas at high temperature self-interstitials become extended and exist in an amorphous bubble form. ${ }^{3,4}$ Recent detailed electronic structure and total-energy calculations of these defects provided a wealth of information, and shed light on the microscopic aspects. ${ }^{5-7}$ From these calculations it is now possible to deduce the formation and migration energies of various charge states. These calculations also reveal various favorable paths of migration in the crystal.

The self-interstitials are expected to form new bonds by weakening the existing bonds. This causes bond distortions that should affect the calculated energy of formation. Since the actual positions of atoms near the defect site are not known, the equilibrium positions can only be obtained by geometry optimizations. In view of the many neighbors involved in the deformation, fully optimized calculations are not feasible. For that reason, in the electronic structure calculations the lattice distortions were either totally omitted, or were limited only to first and second neighboring shells. For example, in the calculations by Car et al. $^{7}$ only the relaxation of nearest neighbors was treated in a self-consistent way, but the longrange lattice relaxations were taken into account by the Keating model. ${ }^{8}$

In the present study we approached this problem from a different direction, and have carried out the molecular dynamic calculations ${ }^{9}$ by using the Stillinger-Weber (SW) model potential. ${ }^{10}$ The premise is that the computer simulations using this potential have been successful in revealing important information regarding the surface and liquid structure of $\mathrm{Si}^{10,11}$ For example, the $\mathrm{SW}$ potential readily leads ${ }^{11}$ to the unbuckled dimer bond formation on $\mathrm{Si}(001)$. Furthermore, it also suggests extended reconstruction of the type proposed by Pandey. ${ }^{12}$ Recent analysis of the $\mathrm{Si}(001)$ surface by scanning tunneling microscopy is confirming these predictions. ${ }^{13}$ The objective of our study is twofold: First is to provide a further understanding on the lattice distortions caused by the self-interstitials. Second is to explore the value of the SW model potential by comparing with the results obtained from the self-consistent field (SCF) total-energy calculations. In the present work employing the SW potential, we found that the lattice relaxations are significant up to a distance of $\sim 11$ a.u. from the defect center, and nonuniform in a given shell. We have alsc calculated the energy of formation for these defects in an ideal (unrelaxed) and relaxed crystal. The energies for the unrelaxed system are found to be larger than one expects from electronic structure calculations. However, upon relaxation these energies are significantly reduced and give values in substantial agreement with the SCF total-energy calculations. ${ }^{7}$ 


\section{METHOD AND MODEL}

The molecular-dynamics simulation technique yields the motion of a given number of atoms governed by their mutual interatomic interactions, this being calculated by numerical integration of Newton's equations of motion. In the traditional molecular dynamics experiment, the total energy $E$ for a fixed number of atoms $N$ in a fixed volume $V$ is conserved as the dynamics of the system evolves in time, and the time average of any property is an approximate measure of the microcanonical ensemble average of that property for a thermodynamic state of $N, V, E$. For certain investigations, it may be advantageous to perform the simulation at constant pressure and/or temperature.

We have chosen an isobaric-isothermal moleculardynamics approach which essentially evolved from experience with the Monte Carlo method. Conventional molecular dynamics consists of integrating Newton's equation of motion to obtain the trajectories of the atoms, where the total energy is a constant of the motion as the system evolves along its trajectory in phase space. In our isobaric-isothermal molecular-dynamics method, we adopt the following two changes from conventional molecular dynamics: (i) In order to simulate a constant temperature, the atomic velocities are renormalized at every time interval $\tau_{T}$, so that the mean kinetic energy corresponds to the given temperature $T$; (ii) in order to simulate a constant pressure, the volume of the computational cell is changed randomly by $\delta V$ within some prescribed range at every time interval $\tau_{P}$, requiring the scaling of all the atomic coordinates by an appropriate factor, and with an accompanying total energy change $\delta U$. Adopting the Metropolis test, if the quantity

$$
\Delta W=\delta U+P \delta V-N k_{B} T \ln (1+\delta V / V)
$$

is negative, this "scaled" configuration is accepted. If it is positive then this configuration is accepted only with the probability equal to $\exp \left(-\Delta W / k_{B} T\right)$. The time evolution of the system is still governed by the numerical integration of the classical equations of motion, but with the velocity renormalization and position scaling being periodically performed at the specified time intervals. To describe this molecular-dynamics method succinctly, the "stochastic dynamics" of the individual atoms in the isobaric-isothermal Monte Carlo method is replaced by the deterministic equations of motion with the added feature of velocity renormalization-everything else remains the same.

We have investigated four different types of selfinterstitials. Tetrahedral-site $\left(I_{T}\right)$ and hexagonal-site $\left(I_{H}\right)$ self-interstitials are located, respectively, at $(a / 2, a / 2, a / 2)$ and $(3 a / 8,5 a / 8,5 a / 8)$. These positions (or equivalent sites related by $T_{d}$ and $D_{3 d}$ point group symmetry) are low charge-density regions in the $\mathrm{Si}$ crystal and have four nearest neighbors for $I_{T}$ and six for $I_{H}$. In Fig. 1 positions of $I_{T}$ and $I_{H}$ together with their neighbors in a conventional cubic cell are illustrated. Since all the defects dealt in this study occur on the $(0 \overline{1} 1)$
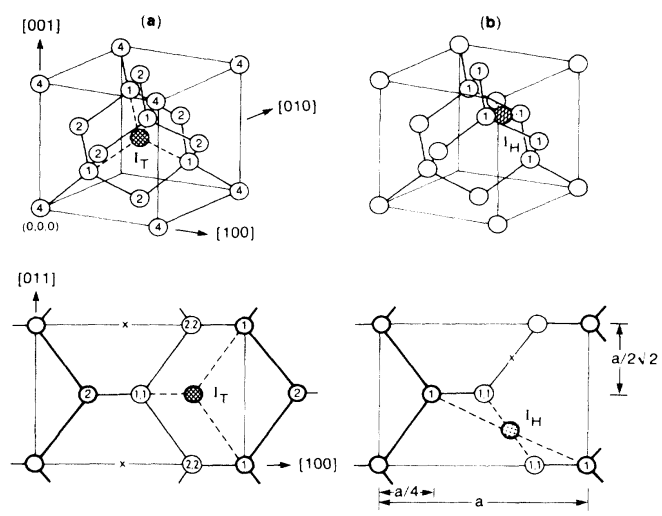

FIG. 1. (a) Tetrahedral-site $\left(I_{T}\right)$ and (b) hexagonal-site $\left(I_{H}\right)$ self-interstitial positions are shown by the shaded circles and crosses in the conventional cubic cell and on the $(0 \overline{1} 1)$ plane. Heavily and lightly outlined circles are the $\mathrm{Si}$ atoms on the $(0 \overline{1} 1)$ plane, and $a / 2 \sqrt{2}$ below, respectively. Numbers in the circles denote the shell. Crosses indicate equivalent interstitial sites and $a=10.26$ a.u. is the lattice constant of Si.

plane, the locations on this plane are also shown in the same figure. The bond-centered interstitial $\left(I_{B}\right)$ is created by breaking one of the $\mathrm{Si}-\mathrm{Si}$ bonds (for example a bond along the [111] direction), and by placing an additional $\mathrm{Si}$ atom at the center. In the split or dumbbell interstitial $\left(I_{D}\right)$, one $\mathrm{Si}$ atom of the perfect crystal located at $(0,0,0)$ is replaced by two $\mathrm{Si}$ atoms at $(-\sqrt{3} a / 8,0,0)$ and $(\sqrt{3} a / 8,0,0)$.

We simulated the crystal having any one of these defects by a periodic supercell consisting of the units of the (011) plane: 5 along [100], 5 along [011], and 16 along the [011] direction. This way, our supercell was formed by 800 host $\mathrm{Si}$ atoms and one interstitial of the type under consideration at the center. The supercell geometry was used to eliminate the edge effects, and at the same time its size is taken sufficiently large to prevent interactions among interstitials in the neighboring supercells.

In view of the fact that the quantum-mechanical totalenergy calculations for geometry optimizations of $\sim 100$ atoms are not feasible yet, much effort has gone into simpler methods. In this context, the potential functions derived from two-body interatomic forces have been used for inert gas solids, but have been found to be inadequate for metals and semiconductors. This failure is remedied by including three-body interactions representing the angular forces. ${ }^{14-16}$ The results obtained so far are encouraging. ${ }^{11,16}$ In fact the ground-state energy of a condensed system of $N$ atoms with respect to the same number of noninteracting atoms may be expressed as a (potential) energy function of nuclear coordinates, $\Phi$ $=\left(R_{1}, R_{2}, \ldots, R_{N}\right)$, within the Born-Oppenheimer approximation. In an equilibrium state at $T=0 \mathrm{~K}$ this energy is equal to the cohesive energy of this system. In general this energy function can be expanded in terms of the pair and three-body interactions assuming that they are, to a first approximation, additive and that the higherorder terms have negligible effects. Usually these interactions are represented by proper functions of the atomic 
TABLE I. Ideal (unrelaxed) and equilibrium (relaxed) atomic positions around a tetrahedral site self-interstitial ( $\left.I_{T}\right)$ on the $(0 \overline{1} 1)$ plane. Here $N$ identifies the shell and $x, y, z$ are coordinates in unit of lattice constant $a$. Also $r_{i}$ and $r_{e}$ are ideal and equilibrium distances from the self-interstitial. The coordinate center is at the $I_{T}$ site, and $\mathbf{x}\|[100], \mathbf{y}\|[011], \mathbf{z} \|[0 \overline{1} 1]$.

\begin{tabular}{|c|c|c|c|c|c|c|c|c|c|}
\hline \multirow[b]{2}{*}{$N$} & \multicolumn{4}{|c|}{ Ideal } & \multicolumn{5}{|c|}{ Equilibrium } \\
\hline & $x$ & $y$ & $z$ & $r_{i}$ & $x$ & $y$ & $z$ & $r_{e}$ & $r_{e}-r_{i}$ \\
\hline 1 & 0.250 & \pm 0.354 & 0.0 & 0.433 & 0.273 & \pm 0.384 & 0.0 & 0.472 & 0.039 \\
\hline 1 & -0.250 & 0.0 & \pm 0.354 & 0.433 & -0.272 & 0.0 & \pm 0.386 & 0.472 & 0.039 \\
\hline 2 & \pm 0.500 & 0.0 & 0.0 & 0.500 & \pm 0.541 & 0.0 & 0.0 & 0.541 & 0.041 \\
\hline 2 & 0.0 & \pm 0.354 & \pm 0.354 & 0.500 & 0.0 & \pm 0.382 & \pm 0.383 & 0.541 & 0.041 \\
\hline 3 & -0.250 & \pm 0.707 & \pm 0.354 & 0.829 & -0.258 & \pm 0.723 & \pm 0.359 & 0.847 & 0.018 \\
\hline 3 & -0.750 & \pm 0.354 & 0.0 & 0.829 & -0.766 & \pm 0.365 & 0.0 & 0.848 & 0.019 \\
\hline 3 & 0.250 & \pm 0.354 & \pm 0.707 & 0.829 & 0.259 & \pm 0.358 & \pm 0.724 & 0.848 & 0.019 \\
\hline 3 & 0.750 & 0.0 & \pm 0.354 & 0.829 & 0.766 & 0.0 & \pm 0.365 & 0.849 & 0.020 \\
\hline 4 & 0.500 & \pm 0.707 & 0.0 & 0.866 & 0.522 & \pm 0.736 & 0.0 & 0.902 & 0.036 \\
\hline 4 & -0.500 & 0.0 & \pm 0.707 & 0.866 & -0.521 & 0.0 & \pm 0.737 & 0.909 & 0.037 \\
\hline 4 & -0.500 & \pm 0.707 & 0.0 & 0.866 & -0.503 & \pm 0.711 & 0.0 & 0.871 & 0.005 \\
\hline 4 & 0.500 & 0.0 & \pm 0.707 & 0.866 & 0.504 & 0.012 & \pm 0.712 & 0.873 & 0.007 \\
\hline 5 & 0.750 & \pm 0.707 & \pm 0.354 & 1.090 & 0.760 & \pm 0.713 & \pm 0.360 & 1.103 & 0.013 \\
\hline 5 & -0.750 & \pm 0.35 & \pm 0.707 & 1.090 & -0.759 & \pm 0.359 & \pm 0.715 & 1.103 & 0.013 \\
\hline 5 & -0.250 & \pm 1.061 & 0.0 & 1.090 & -0.251 & $\mp 1.074$ & 0.0 & 1.103 & 0.013 \\
\hline 5 & -0.250 & \pm 1.061 & 0.0 & 1.090 & 0.251 & $\mp 1.071$ & 0.0 & 1.101 & 0.011 \\
\hline
\end{tabular}

coordinates with certain parameters to be fitted to various equilibrium properties of the matter under consideration. The SW (Ref. 10) model potential for the condensed phases of $\mathrm{Si}$ was constructed in this way, and may be viewed as a generalization of the Keating ${ }^{8}$ potential. It is similar in spirit and form to the pioneering work of Smith $^{17}$ for amorphous $\mathrm{Si}$ and Ge.

Our results are obtained using molecular-dynamics simulation technique, ${ }^{9}$ described above briefly using the SW potential. ${ }^{10}$ The potential is the sum of a combination of pair and triplet potentials, $v_{2}$ and $v_{3}$, scaling by the energy and length scales $\varepsilon$ and $\sigma$ :

$$
\begin{aligned}
& v_{2}=\varepsilon f_{2}\left[\frac{r_{i j}}{\sigma}\right], \\
& v_{3}=\varepsilon f_{3}\left(\frac{\mathbf{r}_{i}}{\sigma}, \frac{\mathbf{r}_{j}}{\sigma}, \frac{\mathbf{r}_{k}}{\sigma}\right),
\end{aligned}
$$

where

$$
f_{2}(r)=\left\{\begin{array}{l}
A\left(B r^{-4}-1\right) \exp \left[\left(r-a_{0}\right)^{-1}\right], \quad r<a_{0} \\
0, \quad r \geq a_{0},
\end{array}\right.
$$

and

$$
\begin{aligned}
f_{3}\left(\mathbf{r}_{i}, \mathbf{r}_{j}, \mathbf{r}_{k}\right)= & h\left(r_{i j}, r_{i k}, \theta_{i}\right)+h\left(r_{j i}, r_{j k}, \theta_{j}\right) \\
& +h\left(r_{k i}, r_{k j}, \theta_{k}\right), \\
h\left(r_{i j}, r_{i k}, \theta_{i}\right)= & \lambda \exp \left[\gamma\left(r_{i j}-a_{0}\right)^{-1}+\gamma\left(r_{i k}-a_{0}\right)^{-1}\right] \\
& \times\left(\cos \theta_{i}+\frac{1}{3}\right)^{2}, \quad r_{i j}, r_{i k}<a_{0} .
\end{aligned}
$$

Otherwise,

$$
h\left(r_{i j}, r_{i k}, \theta_{i}\right)=0,
$$

where $\theta_{i}$ is the angle between atoms $j$ and $k$ subtended at vertex $i$, etc. The SW parameter set is $A=7.049556277$,
$B=0.6022245584, \quad a_{0}=1.80, \quad \lambda=21.0, \quad$ and $\gamma=1.20$. The scaling parameters are $\varepsilon=2.1675 \mathrm{eV}$ and $\sigma=2.0951$ $\AA$. Since these parameters are determined from the crystal as well as from the liquid-state properties, so the range of applicability of this potential is not limited to the tetrahedral coordination. ${ }^{10}$ This point is extremely important for the present study. In our molecular-dynamics calculation, we started with a cluster of 800 atoms in their ideal bulk positions. The additional impurity atom was placed in various sites near the center of this cluster. The atoms are allowed to evolve using molecular dynamics with a very small temperature and to relax to a potentialenergy minimum as the temperature is decreased toward zero and the pressure maintained at zero.

We obtained the energy of formation as follows: First, we calculated the total energy of the perfect periodic supercell. Then each interstitial calculation was carried out in two stages. In the first stage, the crystal atoms and the

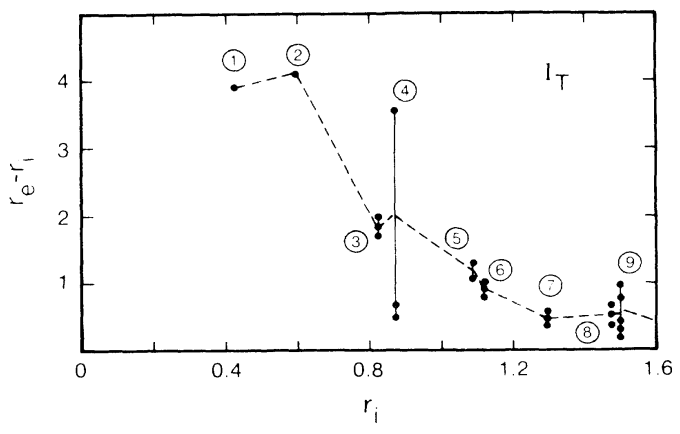

FIG. 2. Displacements (multiplied by 100) of the Si atoms from the tetrahedral-site self-interstitial atom in unit of $a$. The numbers in the circle indicate the shell. $r_{e}$ denotes equilibrium (relaxed); $r_{i}$ denotes ideal (unrelaxed) distances from the selfinterstitial site. 


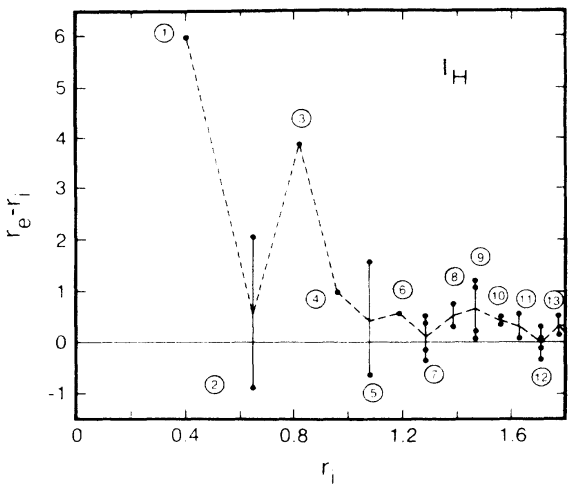

FIG. 3. Displacements (multiplied by 100) of the Si atoms from the hexagonal site self-interstitial atom in units of $a$. See caption to Fig. 2 for other details.

interstitial are frozen at their ideal positions, and their total energies are calculated. In the second stage, both crystal and interstitial atoms are allowed to relax until the equilibrium is reached. The difference between the energy in the equilibrium configuration and the energy of the perfect cell is taken as the formation energy of the defect.

Clearly, no information concerning the electronic structure of the supercell, especially the charge states of interstitial, can be obtained from the present calculations. In this respect, the comparison of the energy of formation obtained from electronic structure calculations with the present results is possible for neutral interstitials only. However, in contrast to the Keating ${ }^{8}$ potential, larger displacements of the crystal atoms can be treated adequately with the present potential.

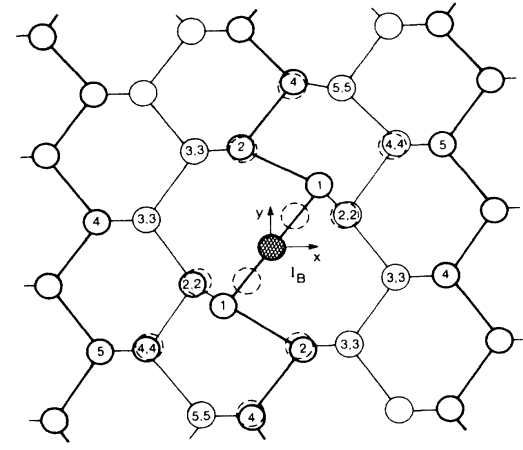

FIG. 4. Equilibrium atomic configuration around a bondcentered self-interstitial $\left(I_{B}\right)$ on the $(0 \overline{1} 1)$ plane. Regular Si atoms on the plane and on the plane below are shown by heavily and lightly outlined circles, respectively. Dashed circles indicate ideal positions (prior to relaxation).

\section{RESULTS AND DISCUSSIONS}

In Table $I$ the positions of the crystal atoms with respect to $I_{T}$ are listed before and after the relaxation. In Fig. 2 the deviations from the ideal defect-host internuclear distance $\left(r_{e}-r_{i}\right)$ are plotted as a function of the ideal (unrelaxed) defect-host atom distance. It is interesting to see that around $I_{T}$ center the second-neighbor atoms have larger displacements than the first-neighbor atoms. Here the maximum relaxation is $0.42 \mathrm{a} . \mathrm{u}$. The relaxations of the atoms at the fourth neighborhood vary between 0.37 and 0.07 a.u., and reflect the directionality of the deformation. As one goes farther away from the defect the displacement decays in an oscillatory fashion. The oscillatory character of the displacements is even

TABLE II. Ideal and equilibrium atomic positions around a hexagonal self-interstitial $\left(I_{H}\right)$ on the $(0 \overline{1} 1)$ plane. See Table I for other details.

\begin{tabular}{|c|c|c|c|c|c|c|c|c|c|}
\hline \multirow[b]{2}{*}{$N$} & \multicolumn{4}{|c|}{ Ideal } & \multicolumn{5}{|c|}{ Equilibrium } \\
\hline & $x$ & $y$ & $z$ & $r_{i}$ & $x$ & $y$ & $z$ & $r_{e}$ & $r_{e}-r_{i}$ \\
\hline 1 & 0.125 & -0.177 & \pm 0.354 & 0.415 & 0.136 & -0.211 & \pm 0.403 & 0.475 & 0.060 \\
\hline 1 & -0.125 & 0.177 & \pm 0.354 & 0.415 & 0.136 & 0.211 & \pm 0.403 & 0.475 & 0.060 \\
\hline 1 & $\mp 0.375$ & \pm 0.177 & 0.0 & 0.415 & $\mp 0.434$ & \pm 0.192 & 0.0 & 0.475 & 0.060 \\
\hline 2 & \pm 0.375 & \pm 0.530 & 0.0 & 0.650 & \pm 0.370 & 0.522 & 0.0 & 0.640 & -0.009 \\
\hline 2 & \pm 0.625 & \pm 0.177 & 0.0 & 0.650 & \pm 0.646 & \pm 0.182 & 0.0 & 0.671 & 0.021 \\
\hline 2 & \pm 0.125 & \pm 0.530 & $\mp 0.354$ & 0.650 & \pm 0.129 & \pm 0.547 & $\mp 0.366$ & 0.671 & 0.021 \\
\hline 3 & 0.375 & -0.177 & \pm 0.707 & 0.820 & 0.396 & -0.180 & \pm 0.740 & 0.859 & 0.039 \\
\hline 3 & -0.375 & 0.177 & \pm 0.707 & 0.820 & -0.396 & 0.180 & \pm 0.740 & 0.859 & 0.039 \\
\hline 3 & \pm 0.625 & $\mp 0.530$ & 0.0 & 0.820 & \pm 0.651 & $\mp 0.560$ & 0.0 & 0.859 & 0.039 \\
\hline 4 & \multicolumn{3}{|c|}{ all four-neighbor atoms } & 0.960 & & & & 0.970 & 0.010 \\
\hline 5 & 0.125 & -0.177 & \pm 1.061 & 1.083 & 0.126 & -0.177 & \pm 1.077 & 1.098 & 0.016 \\
\hline 5 & -0.125 & 0.177 & \pm 1.061 & 1.083 & -0.126 & 0.177 & \pm 1.077 & 1.098 & 0.016 \\
\hline 5 & 0.625 & -0.530 & \pm 0.707 & 1.083 & 0.636 & -0.538 & \pm 0.716 & 1.098 & 0.016 \\
\hline 5 & -0.625 & 0.530 & \pm 0.707 & 1.083 & -0.636 & 0.538 & \pm 0.716 & 1.098 & 0.016 \\
\hline 5 & 0.875 & -0.530 & \pm 0.354 & 1.083 & 0.887 & -0.539 & \pm 0.361 & 1.098 & 0.016 \\
\hline 5 & -0.876 & 0.530 & \pm 0.354 & 1.083 & -0.887 & 0.539 & \pm 0.361 & 1.099 & 0.016 \\
\hline 5 & \pm 0.625 & \pm 0.884 & 0.0 & 1.083 & \pm 0.622 & \pm 0.878 & 0.0 & 1.076 & -0.06 \\
\hline
\end{tabular}


TABLE III. Ideal and equilibrium atomic positions around a bond-centered site self-interstitial ( $\left.I_{B}\right)$. See Table I for other details.

\begin{tabular}{|c|c|c|c|c|c|c|c|c|c|}
\hline \multirow[b]{2}{*}{$N$} & \multicolumn{4}{|c|}{ Ideal } & \multicolumn{5}{|c|}{ Equilibrium } \\
\hline & $x$ & $y$ & $z$ & $r_{i}$ & $x$ & $y$ & $z$ & $r_{e}$ & $r_{e}-r_{i}$ \\
\hline 1 & \pm 0.125 & \pm 0.177 & 0.0 & 0.217 & \pm 0.246 & \pm 0.345 & 0.0 & 0.424 & 0.208 \\
\hline 2 & \pm 0.125 & $\mp 0.530$ & 0.0 & 0.545 & \pm 0.137 & $\mp 0.564$ & 0.0 & 0.581 & 0.036 \\
\hline 2 & \pm 0.375 & \pm 0.177 & \pm 0.354 & 0.545 & \pm 0.401 & \pm 0.187 & $\mp 0.380$ & 0.583 & 0.039 \\
\hline 3 & \pm 0.375 & $\mp 0.530$ & \pm 0.354 & 0.740 & \pm 0.378 & $\mp 0.532$ & \pm 0.358 & 0.745 & 0.005 \\
\hline 3 & \pm 0.125 & \pm 0.177 & \pm 0.707 & 0.740 & \pm 0.124 & \pm 0.177 & -0.713 & 0.746 & 0.006 \\
\hline 3 & \pm 0.625 & $\mp 0.177$ & \pm 0.354 & 0.740 & \pm 0.632 & $\mp 0.178$ & $\mp 0.355$ & 0.747 & 0.007 \\
\hline 4 & \pm 0.125 & $\mp 0.530$ & \pm 0.707 & 0.893 & \pm 0.124 & \pm 0.533 & \pm 0.709 & 0.896 & 0.003 \\
\hline 4 & \pm 0.875 & $\mp 0.177$ & 0.0 & 0.893 & \pm 0.879 & $\mp 0.174$ & 0.0 & 0.896 & 0.003 \\
\hline 4 & \pm 0.125 & \pm 0.884 & 0.0 & 0.893 & \pm 0.133 & \pm 0.910 & 0.0 & 0.919 & 0.026 \\
\hline 4 & \pm 0.625 & \pm 0.530 & $\mp 0.354$ & 0.893 & \pm 0.646 & \pm 0.549 & $\mp 0.363$ & 0.923 & 0.030 \\
\hline 5 & \pm 0.375 & \pm 0.884 & $\mp 0.354$ & 1.023 & \pm 0.382 & \pm 0.895 & $\mp 0.357$ & 1.037 & 0.014 \\
\hline 5 & \pm 0.875 & \pm 0.530 & 0.0 & 1.023 & \pm 0.887 & \pm 0.539 & 0.0 & 1.038 & 0.015 \\
\hline
\end{tabular}

more pronounced in the $I_{H}$ interstitial (see Table II and Fig. 3). The first-neighbor atoms have equal outward displacement by 0.62 a.u. The second-neighbor atoms, however, depending on their relative positions with respect to the defect center, have 0.22 -a.u. expansion or 0.1-a.u. contraction. The third neighbors have quite large displacement by $\sim 0.4$ a.u.

Figure 4 illustrates the equilibrium atomic configuration around the $I_{B}$ defect on the $(0 \overline{1} 1)$ plane. In this case, the two atoms closest to this interstitial are pulled apart by 1.07 a.u. As a result of incorporating an additional Si atom at the bond center, two sevenfold rings are created, causing distortions in the neighboring sixfold rings. For this configuration, one may speculate that the center of the sevenfold ring may provide favorable locations for new interstitials to make the single $I_{B}$ center extended. This way the formation energy of the bond-centered interstitial may even be lowered further. As seen from Table III, the relaxations due to the bond-centered interstitial also exhibit an oscillatory behavior.

The atomic configuration of the dumbbell interstitial is shown in Fig. 5. The two atoms forming the dumbbell ( $D_{1}$ and $D_{2}$ ), have three nearest neighbors each, and thus they form an $s p^{3}$-like bond configuration. In this case also, similar to the bond-centered interstitial two adjacent sevenfold rings are created. The largest relaxation occurs at the fourth neighboring shell (see Table IV). In agreement with the conclusion drawn from earlier calculations, ${ }^{7}$ the atomic relaxations are small for the $T$ site, moderate for the $H$ site, and large for the $B$ site. The main difference is that the relaxations in our work extend over 4-5 coordination shells around the defect. In fact, for all interstitials studied here the relaxation of a crystal atom having an internuclear distance $\left(r_{i}\right)$ from the defect center may be expressed in the following form:

$$
r_{e}-r_{i}=\xi \exp \left(-\alpha r_{i}\right),
$$

where $\xi=0.17, \alpha=2.5$, and $r$ is in units of $a$. In general, up to a distance $r_{i}=a$, relaxation deviates from the above expression and exhibits oscillations with significant amplitudes. However, for $r_{i}>a$ the amplitudes of the oscillations become smaller and relaxations decay exponentially.

The values of the formation energies are given in Table V. The energies for the unrelaxed crystal given in the first column are significantly larger than the values obtained from the SCF total-energy calculations. ${ }^{7}$ In the SCF calculations the electrons are subject to relaxation despite the fact that the atoms are frozen at their ideal positions. It means that even in this unstable structure, electronic charge around the defect center readjusts to lead to a lower energy state. By definition, such an energy lowering should be reproduced by the exact potential function

TABLE IV. Ideal and equilibrium atomic positions around a dumbbell (split) self-interstitial ( $I_{D}$ ). The coordinate center is taken at $D_{1}$ (see Fig. 5) and the distances are given with respect to that center. See Table I for other details.

\begin{tabular}{|c|c|c|c|c|c|c|c|c|c|}
\hline \multirow[b]{2}{*}{$N$} & \multicolumn{4}{|c|}{ Ideal } & \multicolumn{5}{|c|}{ Equilibrium } \\
\hline & $x$ & $y$ & $z$ & $r_{i}$ & $x$ & $y$ & $z$ & $r_{e}$ & $r_{e}-r$ \\
\hline 1 & -0.033 & \pm 0.354 & 0.0 & 0.355 & -0.100 & 0.416 & 0.0 & 0.428 & 0.073 \\
\hline $1\left(D_{2}\right)$ & 0.433 & 0.0 & 0.0 & 0.433 & 0.433 & 0.0 & 0.0 & 0.433 & 0.0 \\
\hline 2 & -0.283 & \pm 0.354 & \pm 0.354 & 0.575 & -0.307 & \pm 0.362 & \pm 0.371 & 0.602 & 0.028 \\
\hline 3 & -0.533 & 0.0 & $\mp 0.354$ & 0.640 & -0.546 & 0.0 & \pm 0.358 & 0.653 & 0.013 \\
\hline 4 & 0.467 & 0.0 & \pm 0.354 & 0.585 & 0.533 & 0.0 & \pm 0.416 & 0.676 & 0.091 \\
\hline 5 & all & 1-neighbc & $\mathrm{ms}$ & 0.740 & & & & 0.746 & 0.006 \\
\hline 6 & -0.783 & 0.0 & 0.0 & 0.783 & -0.791 & 0.0 & 0.0 & 0.791 & 0.007 \\
\hline 7 & -0.033 & \pm 0.354 & \pm 0.707 & 0.791 & -0.035 & -0.356 & -0.709 & 0.794 & 0.003 \\
\hline 8 & 0.717 & \pm 0.354 & \pm 0.354 & 0.874 & 0.740 & \pm 0.370 & \pm 0.363 & 0.904 & 0.030 \\
\hline 9 & 0.467 & \pm 0.707 & \pm 0.354 & 0.918 & 0.468 & \pm 0.708 & \pm 0.356 & 0.921 & 0.003 \\
\hline 10 & -0.533 & \pm 0.707 & \pm 0.354 & 0.954 & -0.547 & \pm 0.717 & \pm 0.359 & 0.971 & 0.017 \\
\hline
\end{tabular}




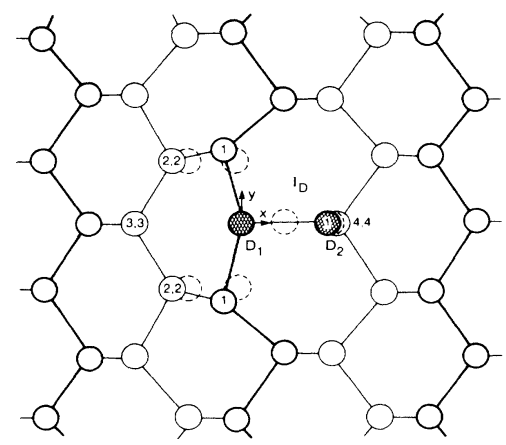

FIG. 5. Equilibrium atomic configuration around a dumbbell (split) self-interstitial ( $\left.I_{D}\right)$ on the $(0 \overline{1} 1)$ plane.

$\Phi$. Since the parameters in the SW model potential are fitted to the equilibrium states, the formation energies of unrelaxed crystal are overestimated. In the case of vacancy where the degree of deviation from the stable configuration is comparably small, the formation energy for the unrelaxed crystal $(\sim 4.34 \mathrm{eV})$ lies within the range of the SCF total-energy values.

The SCF formation energies reported by Car et al. ${ }^{7}$ lie in the $5-8-\mathrm{eV}$ range depending upon the charge state of the impurity and the position of the Fermi level in the energy gap. When Fermi level is in the lower half of the gap, the stable form is a doubly ionized $I_{T}$ interstitial $(\sim 5 \mathrm{eV})$. When Fermi level is in the upper half of the gap the stable forms are neutral $I_{B}$ and $I_{H}$ interstitials with formation enthalpies close to $6 \mathrm{eV}$. Our equilibrium formation energies listed in the second column of Table $\mathrm{V}$ are in reasonable agreement with these values. This demonstrates that the SW potential is capable of giving the correct estimates of the formation energies of interstitials in equilibrium state. The tetrahedral interstitial has the lowest formation energy and thus is predicted to be dominant in low-temperature migration. By contrast, the hexagonal-site interstitial has the highest formation energy, and thus should have lowest equilibrium concentration. The energy difference between $I_{T}$ and $I_{H}$ is found to be $1.6 \mathrm{eV}$. The vacancy formation energy is found to be $4.34 \mathrm{eV}$ with small energy gain upon relaxation. The stability of the vacancy with respect to this formation energy is tested: First, the system is heated up to $1200 \mathrm{~K}$, and then relaxed slowly as the temperature is decreased. This time the system evolved to a different energy minimum with a smaller vacancy volume, suggesting that there exists a manifold of energy minima resulting in different atomic positions around the vacancy. On the other hand, the atomic configuration of the bond centered interstitial as described in Fig. 4 is found to be stable.

It emerges from the high-temperatures self-diffusion data $^{3}$ that the diffusion coefficient may be represented by the relation $D=D_{0} \exp \left(-H / k_{B} T\right)$ with the activation energy $H$ which lies in the range of $4-5 \mathrm{eV}$ depending upon the temperature. In view of the fact that the selfdiffusion activation energy is due almost entirely to the formation energy, the present results confirm the conclusion $^{7}$ that vacancies and self-interstitials mediate the
TABLE V. The formation energies of self-interstitials. Formation energies $E_{f, i}$ are obtained for $\mathrm{Si}$ atoms in their ideal positions; $E_{f, e}$ are obtained for equilibrium positions (after relaxations).

\begin{tabular}{ccc}
\hline \hline Self-interstitial & $E_{f, i}(\mathrm{eV})$ & $E_{f, e}(\mathrm{eV})$ \\
\hline$I_{T}$ & 11.43 & 4.95 \\
$I_{H}$ & 16.02 & 6.54 \\
$I_{B}$ & 90.11 & 5.61 \\
$I_{D}$ & 13.24 & 5.26
\end{tabular}

self-diffusion. The migration path starts from the equilibrium site, and evolves by capturing electrons to overcome the energy barrier. This process is highly dependent on the energies of various charge states and thus on the position of the Fermi level. Since the present model conveys no information about the electronic structure and various charge states of the interstitials we are not able to determine energetically favorable paths, but comparing the energies in Table $\mathrm{V}$ we suggest that the path from $I_{T}$ to $I_{D}$ appears to be most favorable.

Very recently ${ }^{18}$ an important new mechanism for the self-diffusion in Si has been proposed and supported by first-principles total-energy calculations. The mechanism, called concerted exchange, does not require any mediation by defects for atomic diffusion. Instead, an energetically favorable path is found in which the atoms can move through a set of configurations with activation barrier no larger than $4.3 \mathrm{eV}$. Incidentally, this value is very close to the vacancy-formation energy found in our calculation here. The activation energy in the concerned exchange path is certainly competitive with defect-mediated mechanisms and is also consistent with experiments. ${ }^{3}$ It is thus a serious candidate for explaining some significant part of the diffusion in $\mathrm{Si}$. It is interesting that this mechanism involves large displacements of atoms several coordination shells away from the exchange center much like what we are finding around the defect site.

In conclusion, we have shown that with a reasonable potential function such as $\mathrm{SW}$ one is able to obtain results concerning the defect formation energies in a covalent semiconductor in substantial agreement with the SCF calculations. The atomic configurations predicted for the bond-centered and dumbbell interstitials are found to be quite interesting. A study of these geometries by more elaborate methods of total-energy calculations might provide new insights into the energetics of the selfinterstitials. In view of the overestimated formation energies corresponding to unrelaxed crystal it appears that further improvements are required to make the SW potential less repulsive. Works using this type of potential functions are found to be quite useful in suggesting starting configuration for the investigation of large systems, such as large-size surface reconstruction and amorphous state, which seem to be beyond the range of the present SCF techniques.

We acknowledge discussions with Dr. S. T. Pantelides. 
1J. C. Bourgoin and J. W. Corbett, Phys. Lett. 35A, 135 (1972).

${ }^{2}$ G. D. Watkins, J. R. Troxell, and A. P. Chatterjee, in International Conference on Radiation Effects in Semiconductors, Nice, 1978, edited by J. H. Albany, IOP Conference Proceedings No. 46 (IOP, London, 1979).

${ }^{3}$ W. Frank, in Festkörperprobleme: Advances in Solid State Physics, edited by J. Treusch (Vieweg, Braunschweig, 1982), Vol. 21, p. 221.

${ }^{4}$ A. Seeger, H. Föll, and W. Frank, in International Conference on Radiation Effects in Semiconductors, Dubrounik, 1976, edited by N. B. Urli and J. W. Corbett, IOP Conference Proceedings No. 31 (IOP, Bristol, 1976), p. 12.

${ }^{5}$ G. A. Baraff, M. Schlüter, and G. Allan, Phys. Rev. Lett. 50, 739 (1983).

${ }^{6}$ Y. Bar-Yam and J. D. Joannopoulos, Phys. Rev. Lett. 52, 1129 (1984).

${ }^{7}$ R. Car, P. J. Kelly, Atsushi Oshiyama, and S. T. Pantelides, Phys. Rev. Lett. 52, 1813 (1984); S. T. Pantelides, A. Oshiyama, R. Car, and P. J. Kelly, Phys. Rev. B 30, 2260 (1984).

${ }^{8}$ P. N. Keating, Phys. Rev. 145, 637 (1966); I. P. Batra, F. J. Himpsel, P. M. Marcus, R. M. Tromp, M. R. Cook, F. Jona, and H. Liu, in The Structure of Surfaces, edited by M. A. Van Hove and S. Y. Tong (Springer-Verlag, Berlin, 1984), p. 285.

${ }^{9}$ F. F. Abraham, J. Vac. Sci. Technol. B 2, 534 (1984).

${ }^{10}$ F. H. Stillinger and T. A. Weber, Phys. Rev. B 31, 5262 (1985).

${ }^{11}$ F. F. Abraham and I. P. Batra, Surf. Sci. Lett. 163, L752 (1985).

${ }^{12}$ K. C. Pandey, in Proceedings of the 17th International Conference on the Physics of Semiconductors, San Francisco, 1980, edited by D. J. Chadi and W. A. Harrison (Springer-Verlag, Berlin, 1984), p. 55.

${ }^{13}$ R. M. Tromp, R. J. Hamers, and J. E. Demuth, Phys. Rev. Lett. 55, 1303 (1985).

${ }^{14}$ B. M. Axilrod and E. Teller, J. Chem. Phys. 11, 299 (1943).

15J. A. Barker and D. Henderson, Rev. Mod. Phys. 48, 587 (1976), and the references therein.

${ }^{16}$ T. Takai, T. Halicioglu, and W. A. Tiller, Scripta Metallurgica 19, 709 (1985).

${ }^{17}$ D. A. Smith, Phys. Rev. Lett. 54, 815 (1985).

${ }^{18}$ K. C. Pandey, Phys. Rev. Lett. 57, 2287 (1986). 